\title{
MODELING OF COMBUSTION AND PROPULSION PROCESSES OF A NEW CONCEPT GUN USING A GASEOUS PROPELLANT
}

\author{
Ning Liu, Fei Deng, Fei Zhou, Xiangyan Zhang \\ School of Mechanical Engineering, Nanjing University of Science and Technology, Nanjing, China \\ e-mail: liunieng@gmail.com
}

\begin{abstract}
The combustion light gas gun (CLGG) uses a low molecular weight gas as the propellant to burn, expand and propel the projectile out of the barrel with higher muzzle velocities. In order to better understand the interior ballistic process of CLGG, an multidimensional combustion and flow model for CLGG is established. It contains unsteady Reynolds-averaged Navier-Stokes (RANS) equations, the RNG $k-\varepsilon$ two equation turbulence models, and the Eddy-Dissipation Model (EDM) of combustion. Simulation of the interior ballistic process of CLGG is carried out with a propellant of hydrogen and oxygen mixture charged at a particular initial condition. Results show that the spherical front flames spread from the ignition points which divide the flow field as burned and unburned regions in the initial period and expand to the whole flow field subsequently. The filling error of propellants in the chamber will affect the interior ballistic performance, but the impact is relatively small.
\end{abstract}

Keywords: combustion light gas gun, interior ballistics, combustion and propulsion

\section{Introduction}

At present, it is difficult to further improve the performance of conventional guns using the solid propellant. In order to enhance the capabilities of tube weapon, especially the muzzle velocity, many new concept guns have been studied in the world using different kinds of energy resource, such as liquid propellant gun, electrothermal gun and electromagnetic gun. We find that the combustion of solid and liquid propellants produces hot gases with similar components which limit the muzzle velocity of the projectile due to relatively high average molecular weight. Seigel pointed out that if the high muzzle velocity is desired (above $4500 \mathrm{~m} / \mathrm{s}$ ), the choice of the propellant is restricted to either heated hydrogen or heated helium which have low molecular weight at high temperature (Krier and Summerfield, 1979). Therefore, decreasing the molecular weight of the gas in the chamber is a significant method to achieve a higher muzzle velocity in the advanced gun technology research based on chemical energy propulsion. A new species of tube weapon called the combustion light gas gun (CLGG) meets the demand.

A combustion light gas gun uses a low molecular weight combustible gas, such as hydrogen mixed with oxygen, as the propellant. The interior ballistic process for the new concept launching principle can be simply concluded as follows: firstly, the projectile is loaded between the forward end of the gun chamber and the barrel, and then the gun chamber forms a confined space. After carrying out the projectile loading action, the cryogenic temperature fluids, i.e. hydrogen and oxygen propellants, are fed rapidly into the combustion chamber in a liquid or near liquid state using cryogenic feed system before firing. The cryogenic hydrogen and oxygen change to gaseous propellants in the chamber after flowing into the combustion chamber and form the gas mixture in the end. At firing, the gases are ignited by an electrical or laser system. When the mix approaches the ignition point, the reaction increases rapidly and the energy releases due to combustion increasing the pressure in the combustion chamber. When the pressure is 
sufficiently high, the projectile is accelerated by the expansion of hot gases to the muzzle of the exit tube with higher efficiency relative to the solid propellant. The concept has been validated by laboratory experiments and a higher muzzle velocity has been achieved. The principle scheme of CLGG is shown in Fig. 1.

Chamber body Propellant Projectile

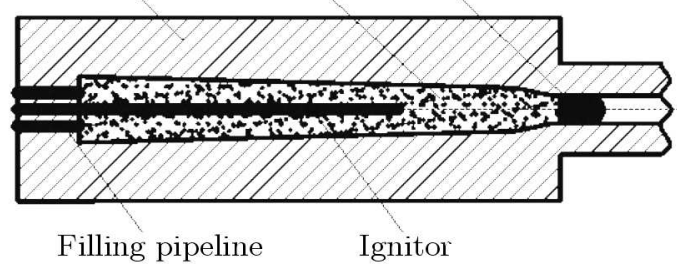

Fig. 1. Schematics of a combustion light gas gun

In fact, the concept of using a column of a low molecular gas to accelerate the projectile was applied in light gas guns (LGG) 50 years ago (Crozier and Hume, 1957). The two-stage light-gas guns are developed subsequently to accelerate projectiles up to $9-10 \mathrm{~km} / \mathrm{s}$ for studies of exterior and terminal ballistics or impact experiments (Angrilli et al., 2003; Munson and May, 1976). In order to further improve the muzzle velocity, the electrothermal light gas gun (ELGG) has been developed which uses electric energy to heat the hydrogen gas in the bore and achieves higher performance than the conventional LGG (Tidman and Massey, 1993). However, the hydrogen gas used in these new species of guns just plays a role of transmission of energy rather than combustion. Actually, hydrogen energy from combustion has been extensively used in many areas (Dunn, 2002), especially in the liquid rocket engines in space program since it has the best energy-to-weight ratio of any fuel (Sutton, 1992; Yeralan et al., 2001). In recent years, the concern for cleaner air has aroused the interest in hydrogen as a vehicular fuel. The hydrogen-fueled internal combustion engines (HICEs) have shown the outstanding characteristics of near-zero emissions and efficiencies in excess of conventional gasoline-fueled ICEs (White et al., 2006). The technology of CLGGs using hydrogen and oxygen as propellants presented here is the latest way of hydrogen application and will promote the performance of guns significantly.

The CLGG has been studied for over ten years (Kruczynski and Massey, 2007). It has been confirmed that the technology provides a minimum of $30 \%$ more muzzle energy than advanced solid propellant guns, which implies significant advantages in the firing range and projectile weight. The $16 \mathrm{~mm}$ CLGG program was firstly conducted by GT Devices and General Dynamics Corp. Previous testing with a $16 \mathrm{~mm}$ CLGG achieved launching 2 gram projectile up to $4200 \mathrm{~m} / \mathrm{s}$ with a hydrogen-oxygen-helium mixture, and $3800 \mathrm{k} / \mathrm{s}$ with methane-oxygen-helium mixtures. The 45mm caliber CLGG program began in 1994 and all tests firings have been conducted by Utron Inc. At present its $155 \mathrm{~mm}$ CLGG has been set up and the firing tests have occurred. The interior ballistic study of CLGG has been carried out gradually in recent years by numerical methods. Liu and Zhang (2011) established a quasi-dimensional interior ballistic model of CLGG which assumed the space in the chamber to be composed of the burned region and the unburned region. Deng et al. (2013) analyzed the influence of the ignition process and the initial condition on interior ballistic characteristics of CLGG by the CFD method.

In the design and development of CLGG, an increase in the interior ballistic prediction capabilities can improve the CLGG hardware reliability and reduce the design and development costs related to experimental activities and firing tests. Both these targets require accurate modeling and numerical simulation as well as reasonable physical understanding of the complex interior ballistics launching phenomenon, which characterize the CLGG behavior and performance during the entire interior ballistic period. This paper is devoted to present the results of the interior ballistics modeling and numerical simulation of CLGG by means of unsteady 
Reynolds-averaged Navier-Stokes (RANS) equations, RNG $k-\varepsilon$ two equation turbulence models, and the Eddy-Dissipation Model (EDM) of combustion.

\section{Model of interior ballistics}

\subsection{Basic assumptions}

The CLGG is focused on providing high velocity projectile launch. It breaks with the traditional solid propellant technology. The combustion process of the premixed gases of the CLGG is complicated. In order to describe the combustion process in the chamber using a mathematical model, the following basic assumptions are proposed for the CLGG system.

(1) The combustion chamber is sealed and no mass leaks during ballistic cycle.

(2) Geometry of the combustion chamber is axisymmetric.

(3) The ignition process is ignored but some energy around the ignition points is taken into acount.

(4) The projectile moves down the barrel under the propulsion of base pressure until it reaches the starting pressure.

(5) The mixing time-scale of hydrogen and oxygen propellants is much larger than the reaction one.

\subsection{Governing equations}

The combustion and flow process in the chamber is a three dimensional unsteady problem. The governing equations for chemically reacting viscous flows are the compressible Navier-Stokes equations with chemical source terms for the mixture composed of gaseous propellant species, which are given as follows:

- continuity equation

$$
\frac{\partial \rho}{\partial t}+\nabla \cdot(\rho \mathbf{u})=0
$$

- momentum equation

$$
\frac{\partial \rho \mathbf{u}}{\partial t}+\nabla \cdot(\rho \mathbf{u u})=-\nabla p+\nabla \cdot\left[\mu\left(\nabla \mathbf{u}+\nabla \mathbf{u}^{\mathrm{T}}\right)\right]+\rho \mathbf{g}+\mathbf{F}
$$

- energy equation

$$
\frac{\partial \rho e}{\partial t}+\nabla \cdot[\mathbf{u}(\rho e+p)]=\nabla \cdot\left(k_{e f f} \nabla T\right)+S_{h}
$$

- species equations

$$
\frac{\partial \rho c_{i}}{\partial t}+\nabla \cdot\left(\rho \mathbf{u} c_{i}\right)=\nabla \cdot\left[D_{i} \nabla\left(\rho c_{i}\right)\right]+S_{i}
$$

where $\rho$ is the total density, $\mathbf{u}$ is the velocity vector, $p$ is the pressure, $T$ is the temperature, $e$ is the total energy per unit mass, $c_{i}$ is the volume concentration of species $i, \mathbf{F}$ is the external force source, $S_{h}$ is the energy source, $s_{i}$ is the mass production rate of species $i$ due to chemical reactions, $k_{e f f}$ is the thermal conductivity which is determined by

$$
k_{e f f}=k_{l}+c_{p} \frac{\mu_{t}}{\operatorname{Pr}}
$$

where $k_{l}$ is the thermal conductivity of the mixture in laminar flow, Pr is the Prandtl constant with a value of 0.71-0.75. 


\subsection{Turbulence model}

Turbulence directly affects the mixing and the combustion process in CLGG. Adequate prediction of turbulence behaviour is necessary for better understanding the CLGG performance. The RNG $k-\varepsilon$ turbulence model derived by Yakhot and Orszag (1986) based on the Renormalization Group theory has been developed to study a number of complex flows. It yields excellent results in cases where the standard $k-\varepsilon$ model predictions are unsatisfactory. The RNG $k-\varepsilon$ model follows the two-equation framework of the turbulent kinetic energy equation and the turbulent energy dissipation rate equation which could be formulated as

$$
\begin{aligned}
& \frac{\partial \rho k}{\partial t}+\nabla \cdot(\rho \mathbf{u} k)=\nabla \cdot\left[\left(\mu+\frac{\mu_{t}}{\sigma_{k}}\right) \nabla k\right]+P_{k}+P_{k b}-\rho \varepsilon \\
& \frac{\partial \rho \varepsilon}{\partial t}+\nabla \cdot(\rho \mathbf{u} \varepsilon)=\nabla \cdot\left[\left(\mu+\frac{\mu_{t}}{\sigma_{\varepsilon}}\right) \nabla \varepsilon\right]+\frac{\varepsilon}{k}\left[C_{\varepsilon 1}\left(P_{k}+P_{\varepsilon b}-C_{\varepsilon 2} \rho \varepsilon\right]\right.
\end{aligned}
$$

where the model constants are given as $C_{\varepsilon 1}=1.42, C_{\varepsilon 2}=1.68 . P_{k}$ is the turbulence production due to viscous forces, which is modeled using

$$
P_{k}=\mu_{t} \nabla \mathbf{u} \cdot\left(\nabla \mathbf{u}+\nabla \mathbf{u}^{\mathrm{T}}\right)-\frac{2}{3} \nabla \cdot \mathbf{u}\left(3 \mu_{t} \nabla \cdot \mathbf{u}+\rho k\right)
$$

$P_{k b}$ and $P_{\varepsilon b}$ represent the influence of the buoyancy forces, which are described below. The buoyancy production term $P_{k b}$ is given as

$$
P_{k b}=-\frac{\mu_{t}}{\rho} g \nabla \rho \quad P_{\varepsilon b}=C_{3} \max \left(0, P_{k b}\right)
$$

where $C_{3}$ is the dissipation coefficient, $C_{3}=1$.

The turbulence viscosity $\mu_{t}$ could be expressed as follows

$$
\mu_{t}=\rho C_{\mu} \frac{k^{2}}{\varepsilon}
$$

where $k$ is the turbulence kinetic energy, $\varepsilon$ is the turbulence energy dissipation rate, and $C_{\mu}$ is the empirical coefficient with the value of 0.0845 .

\subsection{EDM combustion model}

The eddy dissipation model (EDM) is based on the concept that the chemical reaction rate is fast relative to the transport processes, i.e. mixing rates, in the flow. When reactants mix at the molecular level, they instantaneously form products and there is no kinetic control of the reaction process. In turbulent flows, this mixing time is dominated by the eddy properties and the rate is proportional to the mixing time defined by the turbulent kinetic energy $k$ and dissipation $\varepsilon$

$$
\rho \bar{w}_{f u}=\alpha \bar{\rho} \frac{\varepsilon}{k} \min \left(\bar{Y}_{f u}, \frac{\bar{Y}_{o x}}{s}, \beta \frac{\bar{Y}_{p r}}{s+1}\right)
$$

where $\bar{w}_{f u}$ is the average fuel burning rate, $\bar{Y}_{f u}, \bar{Y}_{o x}$ and $\bar{Y}_{p r}$ are average concentrations of the fuel, oxidizer and combustion products, respectively, $\alpha$ and $\beta$ are adjustable parameters.

\subsection{Auxiliary equations}

The auxiliary equations are comprised of the equation of motion of the projectile and the equation of gas state. 
The equation of projectile motion can be written as

$$
\frac{d v_{p}}{d t}=\frac{p_{d} A_{0}}{\varphi_{p} m_{p}}
$$

where $m_{p}$ is the projectile mass, $v_{p}$ is the projectile velocity, $A_{0}$ is the projectile base area, $p_{d}$ is the projectile base pressure, $\varphi_{p}$ is the projectile motion coefficient of second work.

The pressure in the chamber is much higher than that in normal industrial conditions. Therefore, the ideal gas equation is not suitable for the CLGG interior ballistic model. The Abel-Noble equation is applied in the paper, which is widely used in traditional solid propellant guns

$$
p\left(\frac{1}{\rho}-\alpha\right)=R T
$$

where $\alpha$ is the covolume.

\subsection{Boundary condition and numerical method}

For the transient combustion and flow problem, the appropriate initial and boundary conditions that reflect the real physical phenomenon are significant for numerical simulation. For this case, it is supposed that the gaseous propellants are well mixed before firing in the chamber with initial pressure $p_{0}$ and temperature $T_{0}$, as well as chamber volume $V_{0}$. Boundary conditions used in the simulation include no slip boundary for solid walls and moving boundary for the base face of the projectile after moving. The moving grids technique is used in the model to deal with the projectile movement. The governing equations are discretized using an element-based finite volume method. An implicit second order accurate upwind finite volume scheme is used for convection terms while the implicit second order backward Euler scheme for the transient terms. The shape functions following the standard finite element approach are applied for all the diffusion terms.

\section{Results and discussions}

\subsection{Verification of the simulation results}

The experimental data of $45 \mathrm{~mm}$ smooth bore CLGG has been used to compare with the simulation results in order to verify the ability of the model to predict the combustion of gaseous propellants and the main performances of the interior ballistics (Kruczynski and Massey, 2007). The experimental parameters are listed in Table 1. Similar to most of the interior ballistics considerations, we supposed that the ignition occurs when the mixture temperature reaches a specified ignition value by heating the gas along the chamber axis. The simulation results of the muzzle velocity and the maximal pressure at the chamber compared with the reference results are listed in Table 2. The numerical and measured pressure history at the breech end is also shown in Fig. 2 where the experimental curve is shifted to approximately match the time of the calculated maximum pressure, i.e. about $6 \mathrm{~ms}$. Overall, good agreement has been achieved between the model predictions and experimental measurements. We can observe that both the experimental and computed curves present some pressure waves which should be paid special attention to in order to avoid uncontrollable combustion in the chamber. The histories of projectile velocity and oxygen mass fraction are also shown in Figs. 3a and 3b, respectively. It can be observed in Fig. 3b that the oxygen mass fraction is about 0.045 until the projectile reaches the muzzle, i.e. $7.9 \%$ of charged oxygen is not burnt. This phenomenon will be demonstrated in the next Section. 
Table 1. Initial parameters of the $45 \mathrm{~mm}$ CLGG (Kruczynski and Massey, 2007)

\begin{tabular}{|l|c|}
\hline \multicolumn{1}{|c|}{ Parameter } & Value \\
\hline \hline Projectile mass $\left(m_{p}\right)$ & $0.52 \mathrm{~kg}$ \\
\hline Volume of power chamber $\left(V_{0}\right)$ & $5000 \mathrm{ml}$ \\
\hline Total chemical energy of the propellant $(E)$ & $3 \mathrm{MJ}$ \\
\hline Initial pressure $\left(p_{0}\right)$ & $38 \mathrm{MPa}$ \\
\hline Mole ratio between hydrogen and oxygen & $8: 1$ \\
\hline Tube length $(L)$ & $4500 \mathrm{~mm}$ \\
\hline Projectile starting pressure $\left(p_{s}\right)$ & $172.4 \mathrm{MPa}$ \\
\hline Number of ignition points & 5 \\
\hline
\end{tabular}

Table 2. Comparison of experimental and computed results

\begin{tabular}{|l|c|c|}
\hline Parameter & $\begin{array}{c}\text { Maximum pressure } \\
{[\mathrm{MPa}]}\end{array}$ & $\begin{array}{c}\text { Muzzle velocity } \\
{\left[\mathrm{ms}^{-1}\right]}\end{array}$ \\
\hline \hline Experimental value & 225 & 1700 \\
\hline Simulation value & 229 & 1706 \\
\hline
\end{tabular}

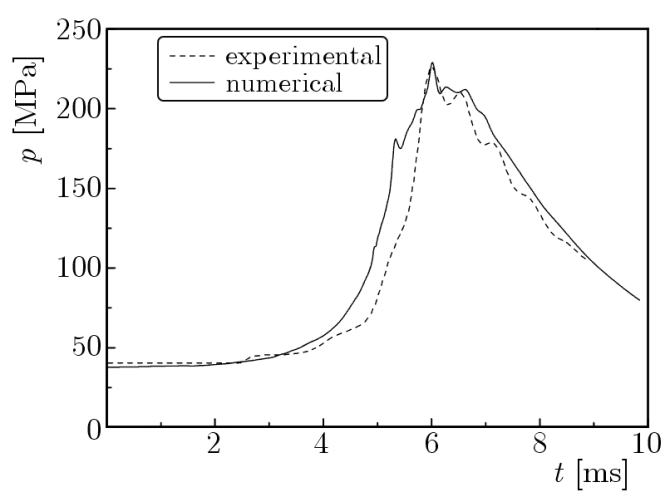

Fig. 2. Comparison between numerical and measured pressure-time traces at the breech end
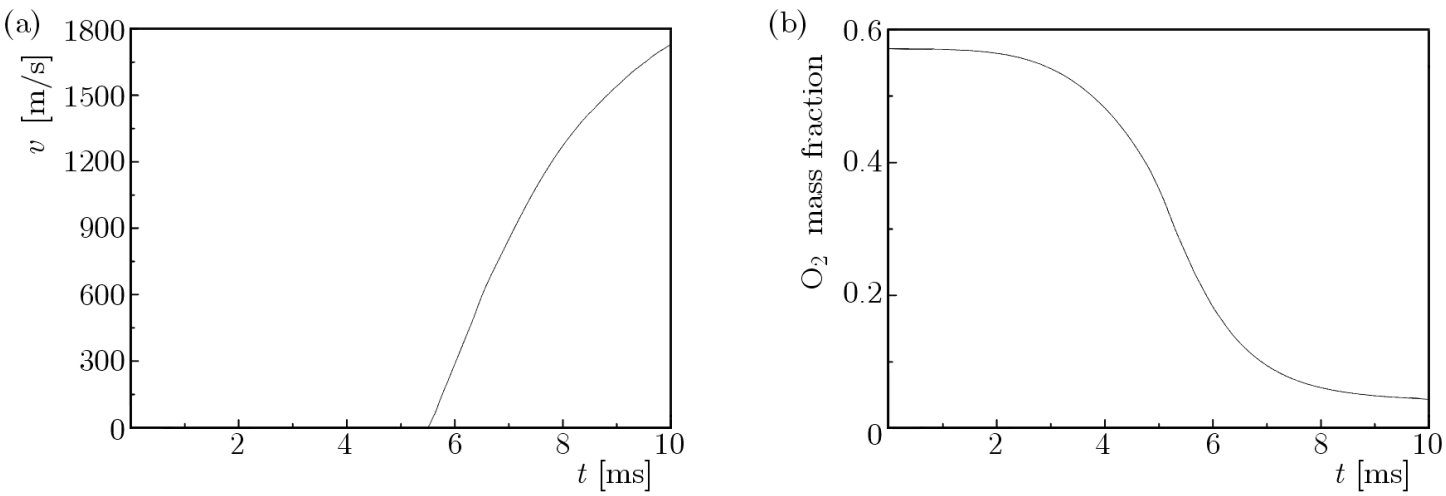

Fig. 3. (a) History of projectile velocity; (b) history of oxygen mass fraction

\subsection{Interior ballistic performance of CLGG}

Figure 4 shows the axial pressure distribution curves in the bore at different times where the endpoints of the curves form the base pressure as a function of the projectile displacement. The pressure difference between the breech end and the projectile base is observed in Fig. 4, and this difference becomes flat after the pressure reaches its peak value. 


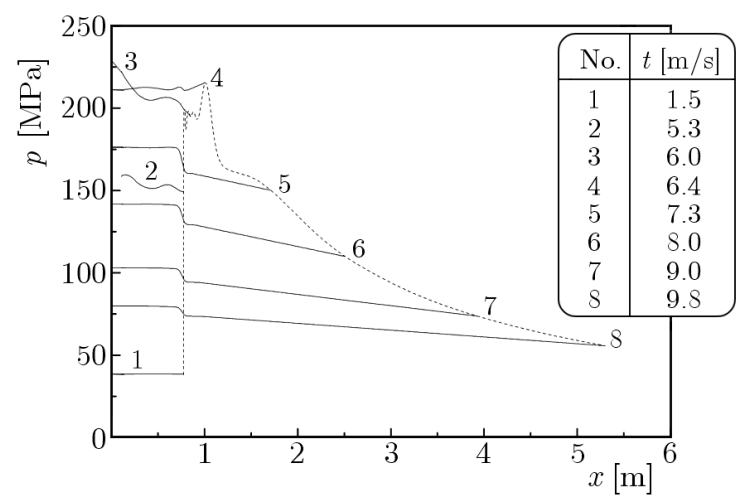

Fig. 4. Pressure distribution on the axis at different time

Figure 5 shows the temperature contours of the gun chamber. The spherical front flame spread from the five ignition points placed on the axis. Therefore, the flow field is divided into burned and unburned regions clearly by the front flame. At about $4.3 \mathrm{~ms}$, the five independent burned regions begin to get in touch with each other and merge into an integral one later. It is interesting to note that the five high temperature cores in the chamber move down the tube following the gas flow after the projectile movement.

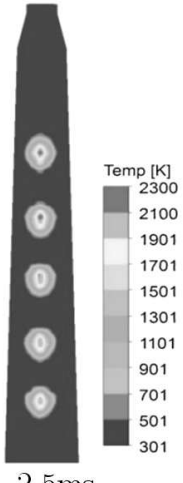

$2.5 \mathrm{~ms}$

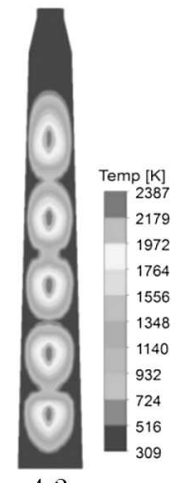

$4.3 \mathrm{~ms}$

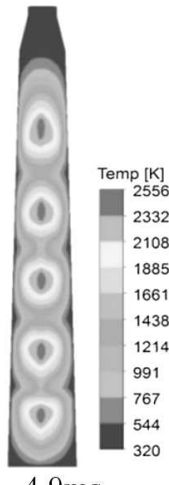

$4.9 \mathrm{~ms}$

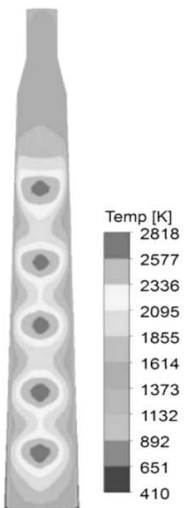

$6.0 \mathrm{~ms}$

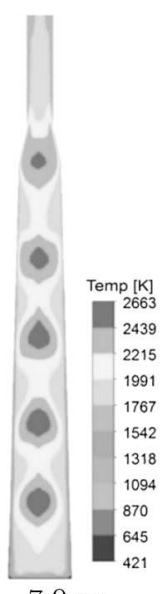

$7.0 \mathrm{~ms}$

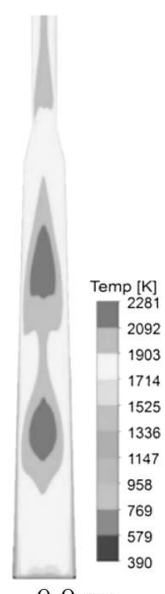

$9.0 \mathrm{~ms}$

Fig. 5. Temperature contours in the gun chamber at different times

The $\mathrm{O}_{2}-\mathrm{H}_{2}$ mixture is filled in the chamber as the propellant rich with hydrogen in order to decrease the average molecular weight and to sufficiently cool the bore surface. Therefore, the oxygen quantity that participates in combustion determines the total chemical energy released during a ballistic cycle and affects the muzzle velocity of the projectile. The oxygen mass fraction distributions at different times are shown in Fig. 6. Same as that seen in Fig. 5, spherical burned regions are clearly visible around the ignition points. The oxygen quantity in the chamber decreases gradually as the burned regions expand until the oxygen burns out in most region of the field. It is important to note that there is still a certain amount of oxygen remained at the breech region until the exit of the projectile through the muzzle. This is considered to be due to weak turbulence at the breech end which leads to incomplete combustion of oxygen using the EDM model.

Figure 7 represents the pressure contours in the chamber. The pressure waves with small amplitude are observed before the pressure increasing to the peak value, whereas the pressure 

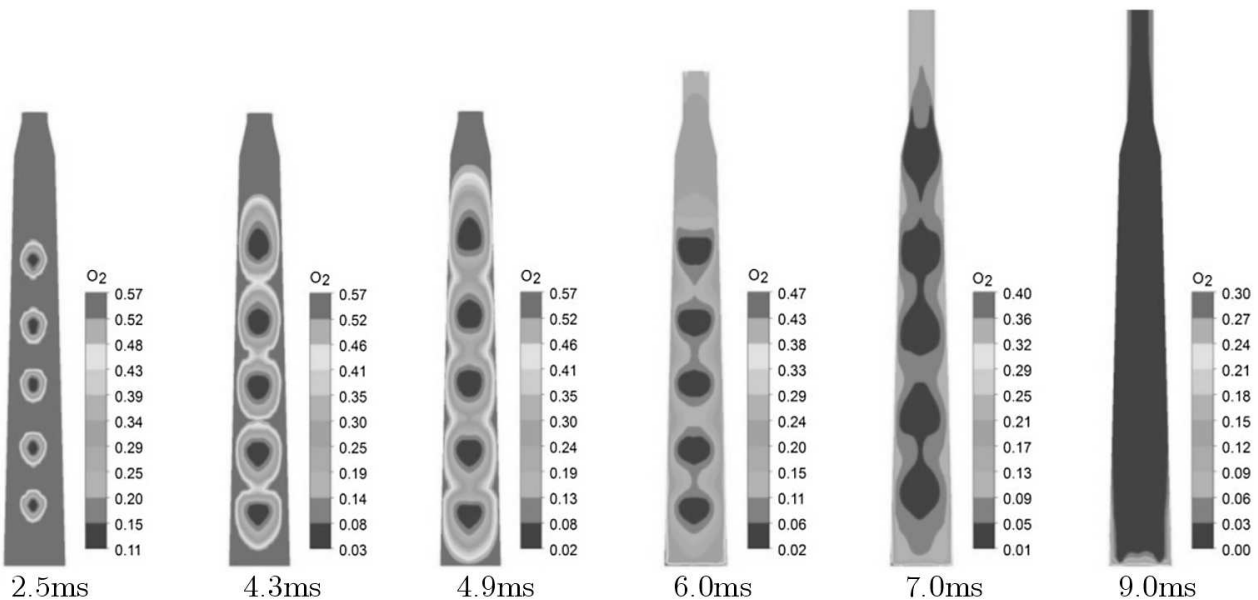

Fig. 6. Distributions of the oxygen mass fraction at different times
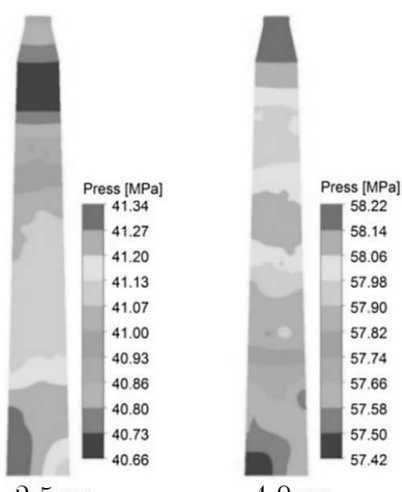

$4.0 \mathrm{~ms}$

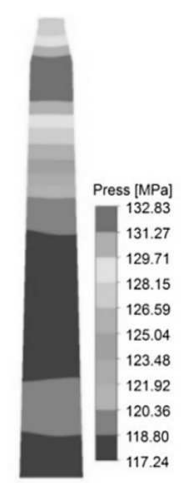

$5.0 \mathrm{~ms}$
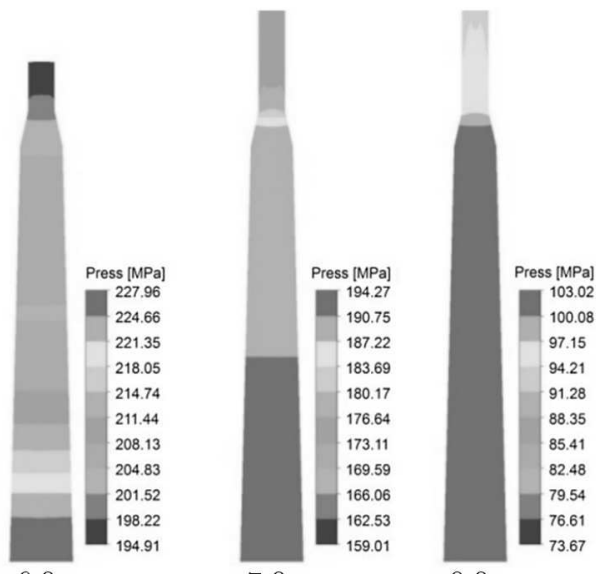

$7.0 \mathrm{~ms}$

Fig. 7. Pressure distributions in the gun chamber at different times

waves disappears subsequently and the pressure decreases gradually from the breech end towards the projectile base after the maximum pressure. We can observe the distribution uniformity of pressure with time in the chamber which is also shown in Fig. 4 where the pressure distributions on the axis are plotted at different times.

\section{Effects of parametric variations on the interior ballistic performance}

\subsection{Effect of the propellant components ratio}

In order to analyze the effect of the propellant components ratio on the interior ballistic performance, we change the initial amount of hydrogen and remain the chemical energy constant. As hydrogen is rich in the chamber, it means that the amount of oxygen should remain constant. The mixture could be written as $n \mathrm{H}_{2}+\mathrm{O}_{2}+2 \mathrm{He}$, and we calculate three cases taking the ratio value $n$ of 4,8 and 12 . The increasing amount of hydrogen raises the initial pressure in the chamber which enhances the maximum pressure and muzzle velocity of the projectile as presented in Figs. 8a and 8b. However, it should be noticed that the temperature significantly decreases as the amount of hydrogen increases in the chamber, as shown in Fig. 8c. It is also worthwhile to point out that for the increasing amount of hydrogen, a longer ignition time is required with the same ignition energy. 

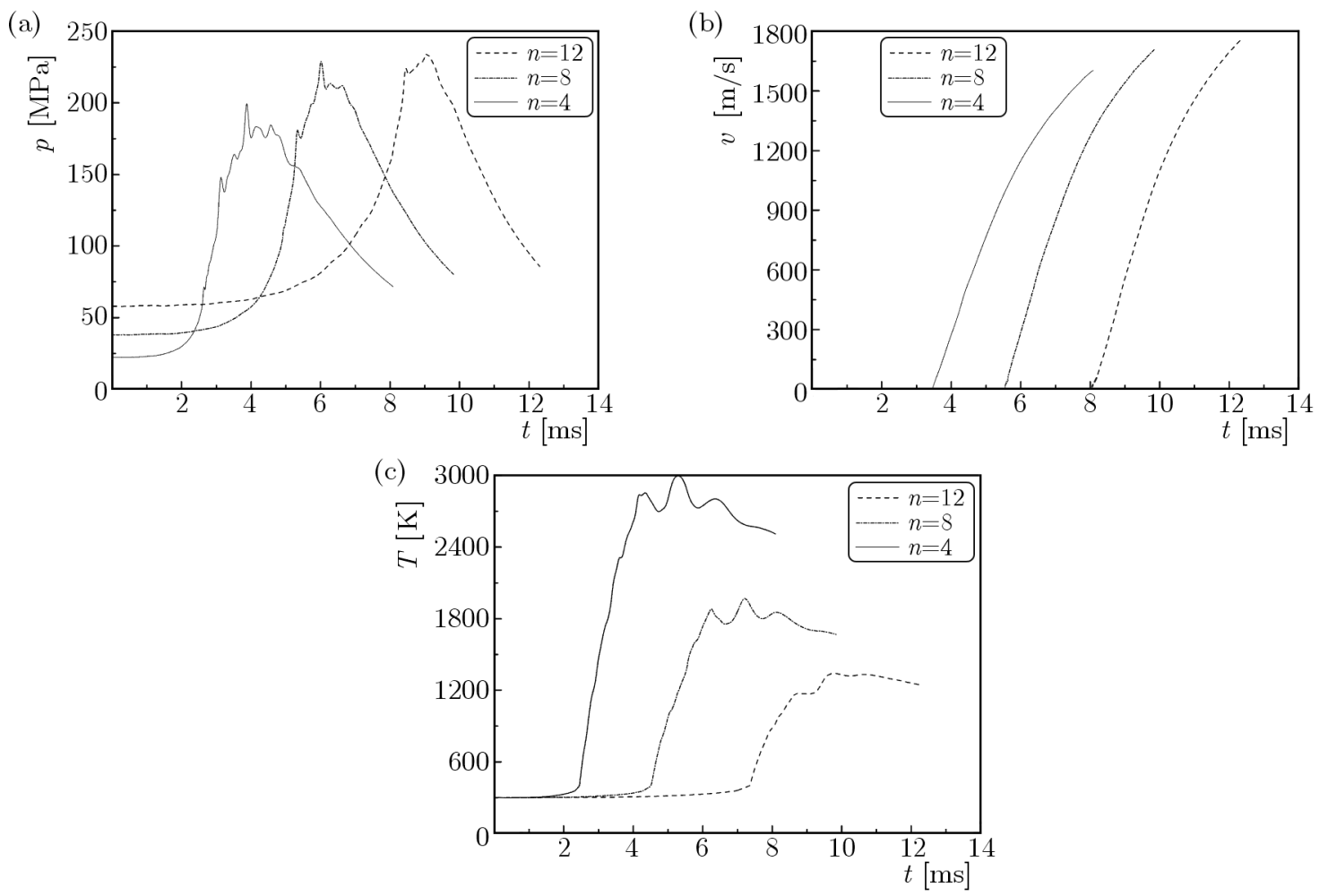

Fig. 8. Predicted pressure-time curves (a), velocity-time curves of the projectile (b) and temperature-time curves (c) for different ratios $n$

\subsection{Effect of the filling accuracy}

In the light of previous research, it has been found that one of the key technologies of CLGG is the filling accuracy control of gaseous propellants. In order to investigate the effect of filling error on the ballistic performance, we repeat the previous calculation while varying the initial amount of oxygen. We calculate the case with oxygen mass relatively increased by $10 \%$. A comparison of pressure curves between the case with the filling error and the accurate one is shown in Fig. 9a.
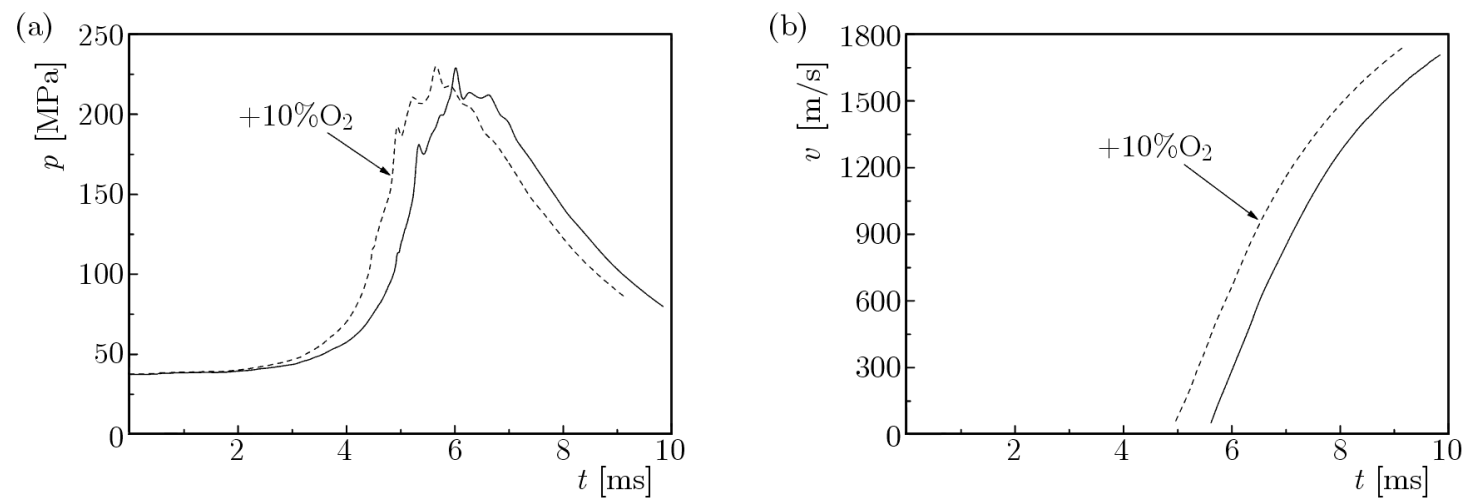

Fig. 9. Comparison of pressure curves (a) and projectile velocity curves (b) with oxygen mass relatively increased by $10 \%$

It can be seen that the increasing of the amount of oxygen increases the pressure raising rate while the other components remain constant. The maximum pressure increases slightly from $229 \mathrm{MPa}$ to $230 \mathrm{MPa}$, i.e. relatively increased by $0.4 \%$. Meanwhile, the muzzle velocity shown in Fig. 9b varies from $1706 \mathrm{~m} / \mathrm{s}$ to $1740 \mathrm{~m} / \mathrm{s}$, i.e. increased by $2 \%$ due to the additional oxygen 
filled in the chamber. Thus, the filling error of propellants in the chamber affects the interior ballistic performance, but the amplitudes are relatively small.

\section{Conclusions}

The development of CLGG aims at providing a long range fire support such as deep strike and effective shore support, other than laboratory instruments. This paper developed a 3D combustion and flow model to study the complex interior ballistic process of CLGG. The numerical simulations based on the CFD method have shown the ability of the model to predict and analyze the main internal ballistics launching phenomenon, which provides a new approach to support and validate the experimental effort. The main conclusions are summarized as follows:

- Good agreement between the predicted and experimental results is obtained except for the ignition process which is of significant interest for future studies.

- The spherical front flames spread from the ignition points which divide the flow field into burned and unburned regions in the initial period and expand to the whole flow field subsequently. This phenomenon may help one to develop the quasi-dimensional interior ballistic model of CLGG.

- The increasing amount of hydrogen enhances the initial pressure, maximum pressure and muzzle velocity of the projectile, while the temperature significantly decreases in the process.

- The filling error of propellants in the chamber affects the interior ballistic performance, but the impact is relatively small.

\section{References}

1. Angrilli F., Pavarin D., De Cecco M., Francesconi A., 2003, Impact facility based upon high frequency two-stage light-gas gun, Acta Astronautica, 53, 3, 185-189

2. Crozier W., Hume W., 1957, High-velocity, light-gas gun, Journal of Applied Physics, 28, 8, $892-894$

3. Deng F., Zhang X.Y., Liu N., 2013, Influences of ignition process and initial conditions on interior ballistic characteristics of combustion light gas gun (in Chinese), Explosion and Shock Waves, 33, 5, 551-555

4. Dunn S., 2002, Hydrogen futures: toward a sustainable energy system, International Journal of Hydrogen Energy, 27, 3, 235-264

5. Hirsch C., 2007, Numerical Computation of Internal and External Flows (second edition): The Fundamentals of Computational Fluid Dynamics, Butterworth-Heinemann, Oxford

6. Krier H., Summerfield M., 1979, Interior ballistics of guns, Progress in Astronautics and Aeronautics, 66, AIAA, New York

7. Kruczynski D., Massey D., 2007, Combustion Light Gas Gun Technology Demonstration, ADA462130

8. LIU N., ZHANG X.Y., 2011, Quasi-dimensional interior ballistic model and numerical simulation of combustion light gas gun, Proceedings of the 26th International Symposium on Ballistics, 414-419

9. Munson D., MaY R., 1976, Interior ballistics of a two-stage light gas gun using velocity interferometry, AIAA Journal, 14, 2, 235-242

10. Sutton G.P., 1992, Rocket Propulsion Elements - an Introduction to the Engineering of Rockets, Wiley-Interscience, New York 
11. Tidman D.A., Massey D.W., 1993, Electrothermal light gas gun, IEEE Transactions on Magnetics, 29, 1, 621-624

12. White C.M., Steeper R.R., Lutz A.E., 2006, The hydrogen-fueled internal combustion engine: a technical review, International Journal of Hydrogen Energy, 31, 10, 1292-1305

13. Yakнot V., Orszag S., 1986, Renormalization group analysis of turbulence. I. Basic theory, Journal of Scientific Computing, 1, 3-51

14. Yeralan S., Pal S., Santoro R.J., 2001, Experimental study of major species and temperature profiles of liquid oxygen/gaseous hydrogen rocket combustion, Journal of Propulsion And Power, 17, 4, 788-793

Manuscript received May 22, 2014; accepted for print October 3, 2014 